\title{
Estimation des réserves en eau souterraine régulatrices dans la partie moyen-atlasique du fleuve Sebou (Maroc)
}

\author{
Sébastien Lebaut ${ }^{1}$, Abdelghani Qadem ${ }^{2}$, Brahim Akdim $^{3}$, Emmanuel Gille $^{1}$, and Mohamed Laaouane ${ }^{3}$ \\ ${ }^{1}$ Unité de recherche LOTERR UR 7304, Université de Lorraine, Metz, France \\ ${ }^{2}$ Laboratoire Dynamique des Paysages, Risques, Patrimoine, \\ Université Sultan Moulay Slimane, Béni Mellal, Maroc \\ ${ }^{3}$ Laboratoire d'Analyses Géo-Environnementales et Aménagement-Développement Durable, \\ Université Sidi Mohamed Ben Abdellah, Fès, Maroc \\ Correspondence: Sébastien Lebaut (sebastien.lebaut@univ-lorraine.fr)
}

Published: 16 November 2021

\begin{abstract}
Résumé. L'estimation de la ressource en eau souterraine dans le Moyen-Atlas est investiguée à partir des débits mesurés dans l'oued Sebou à la station d'Azzaba, sur la période 1959-2015. Pour cela presqu'une centaine de phases de tarissement a été individualisé pour construire des courbes maîtresses de tarissement à partir desquelles le calcul des volumes des nappes est possible. Il est calculé mensuellement à partir du débit modal dont nous posons l'hypothèse qu'il représente la limite entre ruissellement et alimentation uniquement par les nappes. Les calculs donnent un volume de la réserve régulatrice moyen de $54 \mathrm{hm}^{3}$, mais extrêmement variable à l'échelle interannuelle et intra-annuelle. Ces résultats démontrent la faible inertie des aquifères du Moyen Atlas et soulignent la vulnérabilité du secteur agricole vis-à-vis de cette ressource même lors de courte période de sécheresse.
\end{abstract}

The estimate of the groundwater resource in the Middle Atlas is investigated from the runoff measured in the Sebou wadi at the Azzaba station, over the period 1959-2015. Almost a hundred recession curves have been individualized to build the master recession curves from which the calculation of the volumes of groundwater is possible. It was calculated at a monthly scale from the modal flow, which we assume is the limit between runoff and flow supply only by the aquifers. The results indicated a volume of the average regulatory reserve of $54 \mathrm{hm}^{3}$, but extremely variable on an inter-annual and intra-annual scale. These results demonstrate the low inertia of the Middle Atlas aquifers and underline the vulnerability of the agricultural sector to this resource even during short periods of drought.

\section{Introduction}

La notion de ressource en eau souterraine est née de la prise de conscience que des limites s'imposent aux possibilités d'exploitation globales d'une nappe. Dès lors se pose le problème de l'évaluation quantifiée de cette ressource. D'un point de vue théorique les systèmes d'eau souterraine peuvent être classés en trois catégories en fonction de leur profondeur et en fonction de la proximité du réseau drainant (Ophori et Toth, 1990) : peu profond, intermédiaire et régional. Dans le système peu profond, l'eau circule rapidement et répond rapidement à la recharge et décharge, a un temps de trajet court et constitue le débit de base dans les cours d'eau. Ce débit de base est la part de l'écoulement total naturel qui est différé sous l'effet régulateur de divers réservoirs naturels, notamment celui des aquifères à exutoire compris dans le bassin versant et compose tout l'écoulement en période de tarissement (Castany et Margat, 1977). La courbe de tarissement, qui caractérise la décroissance des débits durant cette période, renferme des informations concernant les caractéristiques des aquifères et le stockage (Tallaksen, 1995) et il existe dans la littérature plusieurs modèles conceptualisant les échanges nappe/rivière durant cette période non directement influencées par les pluies (Zécharias et Brutsaer, 1988). 
Il est donc possible de calculer un volume dynamique $V_{\mathrm{d}}$ de la nappe dans un bassin versant (Sanz et Recio, 2011) qui correspond à la notion de réserve régulatrice proposée par Castany (1968). Tout l'enjeu de cette quantification à partir des hydrogrammes journaliers réside dans la bonne formulation du tarissement et dans la séparation de celui-ci en débit d'écoulement et débit de base. Dans cette communication nous présentons une démarche méthodologique pour apprécier la réserve régulatrice dans le bassin du haut Sebou à partir de l'hydrogramme de la station hydrométrique d'Azzaba verrouillant le bassin versant du Sebou $\left(4677 \mathrm{~km}^{2}\right)$ à sa sortie du Moyen-Atlas. A notre connaissance, s'il existe de nombreuses estimations de volumes de nappe à partir de sources dans les basaltes ou karstiques, aucune évaluation sur l'ensemble du bassin n'est disponible.

\section{Secteur d'étude}

Le bassin versant du Haut Sebou s'inscrit exclusivement dans le Moyen-Atlas (Fig. 1). Du point de vue géologique le Moyen-Atlas appartient au système intracontinental atlasique qui traverse l'Afrique du Nord d'ouest en est, de l'Atlantique jusqu'à la Méditerranée centrale selon le parallèle $34^{\circ}$ de latitude nord. Il en constitue une branche qui traverse en oblique le domaine des mesetas avec des altitudes qui dépasse les $3000 \mathrm{~m}$ (Michard et al., 2008). Le MoyenAtlas central s'inscrit dans deux unités morphostructurales. A l'ouest, surplombant la meseta ouest c'est le Moyen-Atlas tabulaire : causse établit dans des séquences sédimentaires carbonatés du Jurassique inférieur (Trias, Lias) et jalonné d'appareils volcaniques et de coulées basaltiques datant du Cénozoïque (Frizon de Lamotte et al., 2008) dont les altitudes sont de l'ordre de $1200 \mathrm{~m}$. La partie est correspond au Moyen-Atlas plissé : bordé par deux failles majeures de direction NE-SO, il correspond à une succession de cuvettes synclinales dans lesquelles des marnes affleurent et de lignes de crêtes anticlinales dans des dolomies correspondant à un relief de montagne dont le point culminant est de $3081 \mathrm{~m}$ (Jebel Bou Iblane).

La principale caractéristique du Moyen-Atlas central est l'empreinte du calcaire et des phénomènes karstiques (De Waele et Melis, 2009). Le fonctionnement hydrologique de l'hydrosystème découle bien évidemment de cette donne géologique (Fig. 2, Table 1). Le trait dominant de l'hydrosystème est l'infiltration généralisée et la circulation souterraine de l'eau dans le substratum très perméable qui alimente les résurgences en pied de versants au contact de formations imperméables (Amraoui et al., 2003).

Le contexte climatique favorable aux forts abats pluviométriques de la région, $523 \mathrm{~mm}$ en moyenne annuelle sur la période 1974/2010 (Qadem, 2015), couplé à une lithologie générale favorable à la formation d'aquifère induit de fortes potentialités de ressource en eau, néanmoins extrêmement variables.

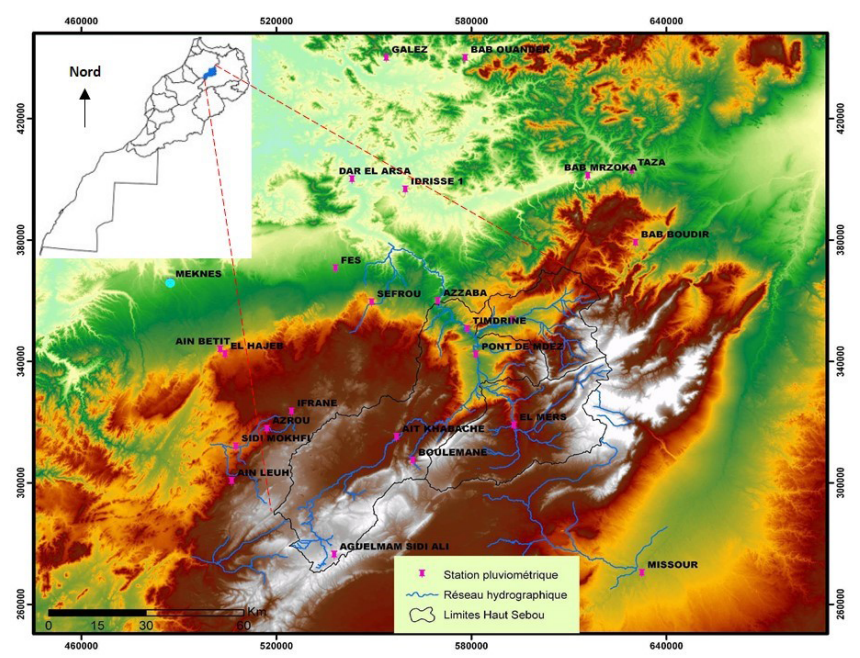

Figure 1. Localisation du bassin du Haut-Sebou.

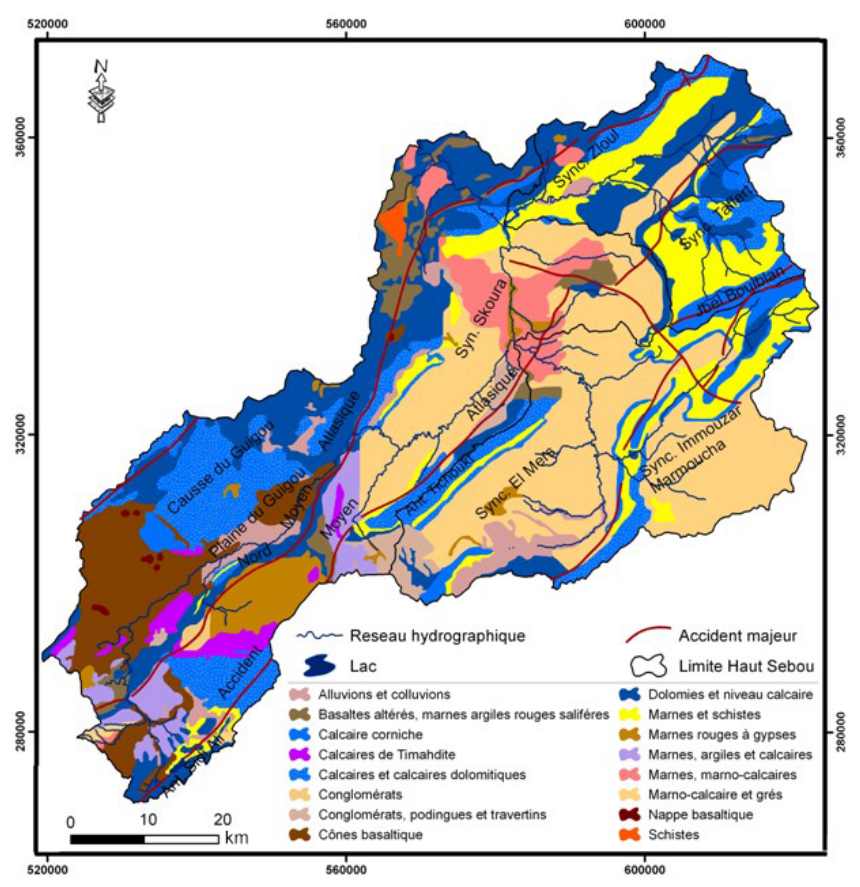

Figure 2. Lithologie du bassin du Haut-Sebou.

\section{Données et méthode}

\subsection{Données}

Les données hydrologiques à la station d'Azzaba $\left(4677 \mathrm{~km}^{2}\right)$ (Fig. 1) sont produites par l'Agence du Bassin Hydraulique du Sebou (ABHS). Disponibles de 1959 à 2015, elles sont réputées de bonnes qualités. 
Tableau 1. Synthèse lithologie/perméabilité dans le bassin versant du Haut Sebou.

\begin{tabular}{llrr}
\hline Roches dominantes & Perméabilité & Surface $\left(\mathrm{km}^{2}\right)$ & $\%$ \\
\hline Alluvions et colluvions & Perméable & 238 & 5 \\
Basaltes, poudingues, conglomérats, calcaires et dolomies & Perméable & 2099 & 45 \\
Alternances de marnes, calcaires et grés & Semi-perméable & 1591 & 34 \\
Marnes, argiles et schistes & Peu perméable & 752 & 16 \\
\hline
\end{tabular}

\subsection{Méthode}

La réserve régulatrice correspond au volume d'eau renouvelable dans les conditions climatiques de la période considérée. Ce dernier peut être apprécié en s'appuyant sur les phases de tarissement des cours d'eau, étant entendu qu'elles représentent la vidange de la réserve régulatrice par les sources et le drainage des cours d'eau. Dans ce cas $V=Q 0 / \alpha$ avec $Q 0$, le débit au début du tarissement et $\alpha$ le coefficient de tarissement.

D'application relativement simple sur les sources, son application sur les cours d'eau est beaucoup plus délicate à mettre en œuvre : choix du débit $Q 0$ à partir duquel calculer le volume, calcul de $\alpha$. Nous proposons d'estimer les volumes mensuels d'eau souterraine constituant la réserve régulatrice des aquifères du bassin du Haut-Sebou sur la base du débit modal pour $Q 0$ et le calcul d'un coefficient de tarissement.

\subsubsection{Détermination du débit modal}

A la suite de plusieurs auteurs, Gamez et Zumstein (1993), font l'hypothèse que dans une série de Qmj classés, la limite entre débits causés par le ruissellement et débits induits uniquement par la vidange des nappes est le débit modal. Compte tenu d'une distribution dissymétrique des Qmj les auteurs calculent le mode à partir d'une loi de Fréchet, loi de Gumbel à laquelle on substitut les $Q$ à $\log Q$, suivant :

$\log (Q j)=\log \left(Q j_{0}\right)-\frac{1}{K} \log [-\log (F)]$

Avec :

$\log \left(Q j_{0}\right)=$ mode $=\overline{\log (Q j)}-0,45 S[\log (Q j)]$

$\frac{1}{K}=$ gradex de Fréchet $=0,78 S[\log (Q j)]$

$S[\log (Q j]=$ écart type de la distribution des logarithmes naturels des débits. $\overline{\log (Q j)}=$ moyenne des logarithmes naturels des débits.

\subsubsection{Calcul du coefficient de tarissement}

Les phases de tarissement sont des événements courts qui varient l'un par rapport à l'autre en fonction des variations de facteurs physiques (Moore, 1997) tel que l'évapotranspiration, l'inégale distribution des pluies, la présence de plusieurs aquifères.... Ainsi l'hétérogénéité lithologique du milieu d'application de cette expression linéaire a comme effet une variabilité temporelle du tarissement. Afin de pallier celle-ci et d'aboutir à un coefficient de tarissement moyen nous avons construit une « courbe maittresse de tarissement» pour laquelle l'ensemble des phases de tarissement définies à partir des chroniques de débits moyens journaliers est utilisé simultanément. Deux méthodes sont utilisées pour l'évaluation : corrélation et matching strip.

- Méthode des corrélations : déterminer la pente moyenne d'un épisode de tarissement particulier, soit pour calculer un rapport $\alpha t$ en corrélant les débits $Q 0$ et $Q$ à $t$ jours $\left(Q_{t}\right)$, pour chaque phase observée (Tallaksen et Van Lanen, 2004). Le principe est d'organiser le nuage de points $\left(Q_{t}, Q_{t}+\mathrm{d} t\right)$ de manière à dessiner une droite de régressions, dont le coefficient de tarissement $\alpha$ est exprimé par la relation suivante :

$$
k=\exp (-\alpha)=\left(\frac{Q}{Q 0}\right)^{1 / t}
$$

- Méthode «matching strip » (MSM) : à partir des débits de la phase de tarissement exprimés en ln de manière à former une droite, la méthode consiste ensuite à lier les différents épisodes de tarissement afin qu'ils forment une seule droite (Toebes and Strang, 1964).

\section{Résultats et discussion}

Sur la période considérée, 98 phases de tarissements supérieures à 5 jours ont été individualisées pour une durée moyenne de 14.6 jours. La méthode des corrélations renvoie un coefficient $\alpha$ de $1,2 \times 10^{-2}$ (Fig. 3), ce qui est en accord avec des formations aquifères à "perméabilité de fissures ». La méthode MSM (Fig. 4) permet de nuancer cette valeur en fonction des saisons et donc en fonction du remplissage des aquifères. Les valeurs $\alpha$ sont comprises entre $1,7 \times 10^{-2}$ au printemps avec un remplissage maximum et $1 \times 10^{-2}$ en hiver (Fig. 4). En outre, le calcul du coefficient sur 4 longues phases de tarissement de 80 à 100 jours pour des débits $Q 0$ compris entre 6,8 et $9,1 \mathrm{~m}^{3} / \mathrm{s}$ a fourni des résultats compris entre $1,1 \times 10^{-2}$ et $1,3 \times 10^{-2}$. Afin de tenir compte de cette variabilité, le volume dynamique $V_{\mathrm{d}}$ a été calculé selon 3 

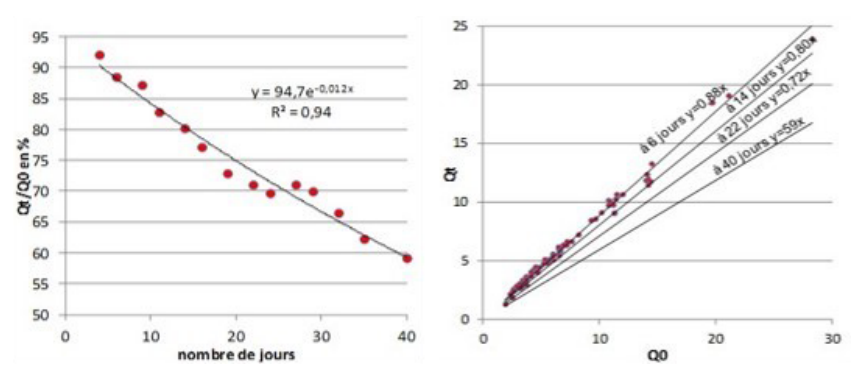

Figure 3. Méthode des correlations.
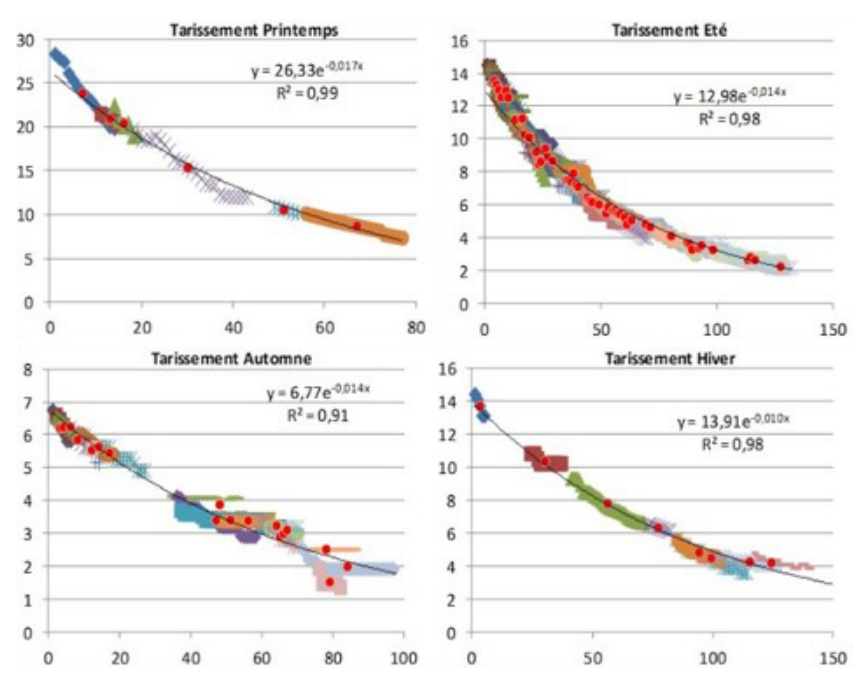

Figure 4. Méthode MSM.

intervalles de débit $Q 0$ : supérieur à $20 \mathrm{~m}^{3} / \mathrm{s}$ avec un $\alpha$ de $1710^{-2}$, compris entre la valeur précédente et $5 \mathrm{~m}^{3} / \mathrm{s}$ avec un $\alpha$ de $1,3 \times 10^{-2}$ et inférieur à la valeur précédente un $\alpha$ de $1 \times 10^{-2}$.

Les débits modaux mensuels ont été calculés sur trois années glissantes afin d'avoir un nombre de valeurs suffisant pour appliquer la méthode de détermination du débit modal (Fig. 5).

Les méthodes de segmentation des longues séries appliquées aux précipitations caractéristiques du secteurs (station de Sefrou, représentative des pluies dans le Haut Sebou) mettent en évidence 3 périodes homogènes : 1959/1980, 1981/2008, 2009/2015. En moyenne la ressource régulatrice en eau souterraine est de $80 \mathrm{hm}^{3}$, mais elle est extrêmement variable (Table 2). Dans la période sèche 1981-2008 elle n'était que de $54 \mathrm{hm}^{3}$, en moyenne, soit deux fois inférieures à celle de la période humide 1959-1980. Les contrastes sont davantage accusés pour les débits minimums, généralement en juillet, avec pour les années particulières de la moitié des années 1990 une ressource de l'ordre de $1 \mathrm{hm}^{3}$ seulement. Les chiffres pour la période récente sont relativement proches de ceux de la période humide. Notons également que les nappes contenues dans le système aquifère du haut Sebou sont très peu inertielles puisque le rapport entre le volume

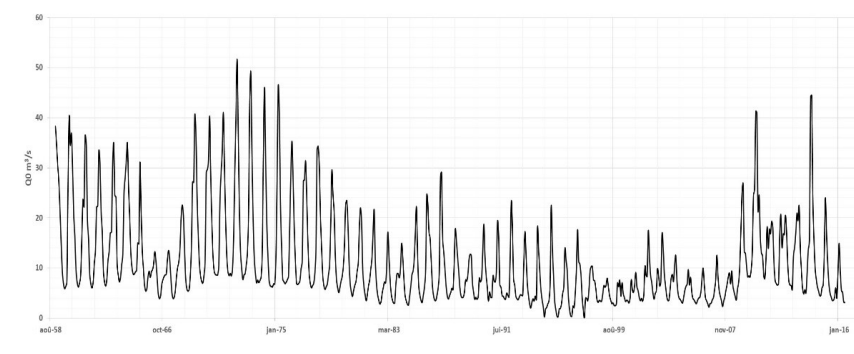

Figure 5. Chronique des débits modaux $(Q 0)$ moyens glissant sur 3 années à la station d'Azzaba, 1960-2015.

Tableau 2. Synthèse sur la ressource en eau dans le bassin du Haut Sebou.

\begin{tabular}{llrrrr}
\hline & 1959-2015 & 1959-1980 & 1981-2008 & 2009-2015 \\
\hline \multicolumn{1}{l}{ Moyenne } & 80 & 108 & 54 & 97 \\
\hline Max & Moyenne & 160 & 212 & 113 & 170 \\
& Min & 59 & 95 & 59 & 106 \\
& Max & 298 & 298 & 183 & 261 \\
& Ecart-type & 53 & 38 & 28 & 42 \\
& Coeff. Var. & $33 \%$ & $18 \%$ & $25 \%$ & $24 \%$ \\
\hline \multirow{2}{*}{ Min } & Moyenne & 35 & 49 & 22 & 42 \\
& Min & 1 & 31 & 1 & 24 \\
& Max & 66 & 66 & 33 & 64 \\
& Ecart-type & 13 & 8 & 6 & 11 \\
Coeff. Var. & $38 \%$ & $16 \%$ & $27 \%$ & $27 \%$ \\
\hline
\end{tabular}

maximum et minimum annuel est de 1 à 4 en moyenne mais peut être supérieur à 10 sur des années particulières, comme en 1995 par exemple (Fig. 6).

La principale limite à nos résultats est liée à la nature des données utilisées. En effet, les débits sont mesurés à la station d'Azzaba qui est située à l'aval d'un réseau hydrographique principal de $262 \mathrm{~km}$, le long duquel des prélèvements sont réalisés pour irriguer des cultures. Les débits soustraits minorent donc les débits à la station hydrométrique et donc les débits modaux à partir desquels sont calculés les volumes dynamiques $V_{\mathrm{d}}$. La nature karstique des aquifères, impliquant des transferts d'eau du bassin du haut Sebou vers les bassins contigus (Akdim et al., 2012) et notamment vers l'Oum Er Rbia situé plus au sud, ne permet d'établir un bilan des nappes à partir de nos valeurs.

\section{Conclusions}

La démarche méthodologique mise en œuvre sur le bassin amont du Haut Sebou permet de quantifier les réserves en eau souterraine d'une fraction du Moyen-Atlas. Dans les limites du bassin versant fonctionnel, de $80 \mathrm{hm}^{3}$ en moyenne sur la période 1959/2015, les valeurs comprises entre 298 et $0.5 \mathrm{hm}^{3}$ montrent une variabilité extrême de cette ressource en eau en accord avec la nature calcaire et basaltique des aquifères. Ceci démontre que les nappes du «château d'eau du Maroc » sont très peu inertielles pouvant entraîner conjoncturellement de très faibles volumes d'eau dispo- 


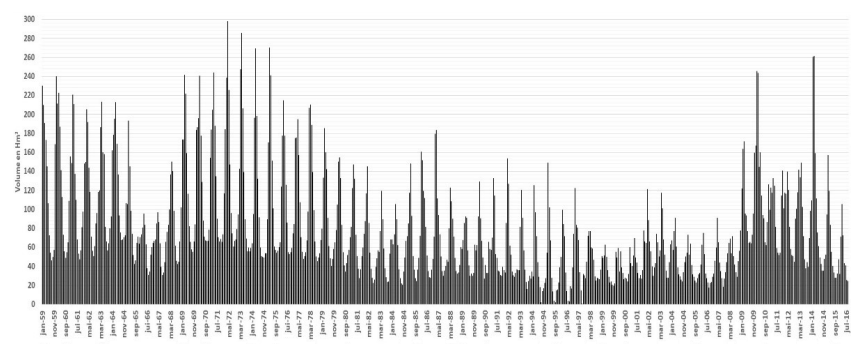

Figure 6. Volumes mensuels de la réserve régulatrice dans le bassin du Haut Sebou (1959-2015).

nibles en surface pour l'irrigation des parcelles agricoles via le système d'eau gravitaire traditionnel. A cet égard, les années 1980/90, marquées par une sécheresse pluviométrique sévère, ont vu cette ressource chuter de moitié par rapport à la période $1960 / 70\left(54 \mathrm{hm}^{3}\right.$ contre $\left.108 \mathrm{hm}^{3}\right)$ et ont été marquées par de profond changement hydro-agricole. Il sera intéressant d'étendre cette quantification des ressources en eau souterraine à l'ensemble du Moyen-Atlas en s'appuyant sur les débits des autres fleuves y prenant leur source comme l'Oum Er Rbia ou la Moulouya.

Disponibilité des données. Les données de débits ont été fournies par l'agence du bassin hydraulique du Sebou (ABHS) de Fès.

Collaborateurs. Cet article présente une partie des résultats d'un projet visant à quantifier et à spatialiser la ressource en eau dans le bassin du Haut Sebou. SL, BA, EG et ML ont participé à la conceptualisation du projet et à la recherche de son financement. Plus spécifiquement pour cet article SL et AQ ont contribué à élaborer la méthodologie, à analyser les données et à la rédaction.

Intérêts concurrents. Les auteurs déclarent qu'ils n'ont aucun conflit d'intérêts.

Clause de non-responsabilité. Publisher's note : Copernicus Publications remains neutral with regard to jurisdictional claims in published maps and institutional affiliations.

Déclaration du numéro spécial. This article is part of the special issue "Hydrology of Large River Basins of Africa". It is a result of the 4th International Conference on the "Hydrology of the Great Rivers of Africa", Cotonou, Benin, 13-20 November 2021.

Remerciements. Ce travail s'inscrit dans l'Action Intégrée franco-marocaine no. MA 10-244 d'un Partenariat Hubert Curien.

\section{Références}

Akdim, B., Sabaoui, A., Amyay, Mh., Laaouane, M., Gille, E., and Obda, K. : Influences hydro karstiques du système sourcier Aïn Sebou-Timedrine-Ouamender Sur l'hydrologie de l'oued Sebou (Moyen Atlas, Maroc), Z. Geomorph., 56, 165-181, 2012.

Amraoui, F., Razack, M., and Bouchaou, L. : Turbidity dynamics in karstic systems. Example of Ribaa and Bittit springs in the Middle Atlas (Morocco), Hydrolog. Sci. J., 48, 971-984, 2003.

Castany, G. : Prospection et exploitation des eaux souterraines. Paris, Dunod édition, 718 pp., 1968.

Castany, G. et Margat, J. : Dictionnaire français d'hydrogéologie, BRGM Service géologique national, Orléans, 249 pp., 1977.

De Waele, J. and Melis, M. T. : Geomorphology and geomorphological heritage of the Ifrane-Azrou region (Middle Atlas, Morocco), Environ. Geol., 58, 587-599, 2009.

Frizon de Lamotte, D., Zizi, M., Missenard, Y., Hafid, M., El Azzouri, M., Maury, R. C., Charrière, A., Taki, Z., Benammi, M., and Michard, A. : The Atlas system - Continental Evolution : The geology of Morocco, Lecture notes in earth sciences 116, Springer-Verlag Berlin Heidelberg, 2008.

Gamez, P. and Zumstein, J. F. : Méthode de détermination des débits de base et de ruissellement d'un cours d'eau, L'eau, la terre et les hommes - Hommage à René Frécaut, 113-120, Presse Universitaire de Nancy, 1993.

Michard, A., Frizon de Lamotte, D., Saddiqi, O., and Chalouan, A. : An outline of the geology of Morocco - Continental Evolution : The geology of Morocco, Lecture notes in earth sciences 116, Springer-Verlag Berlin Heidelberg, 2008.

Moore, R. D. : Storage-outflow modelling of streamflow recessions, with application to a shallow-soil forested cathment, J. Hydrol., 198, 260-270, 1997.

Ophori, D. and Toth, J. : Relationships in regional ground-water discharge to stream : an analysis by numerical simulation, J. Hydrol., 119, 215-244, 1990.

Qadem, A. : Quantification, modélisation et gestion de la ressource en eau dans le bassin versant du Haut Sebou (Maroc), Thèse de doctorat, Université de Lorraine et Fès, 272 pp., 2015.

Sanz, E. et Recio, B. : A method to assess annual average renewable groundwater reserves for large regions in Spain, Hydrolog. Sci. J., 56, 99-107, 2011.

Tallaksen, L. M. : A review of base flow recession analysis, J. Hydrol., 165, 359-370, 1995.

Tallaksen, L. M. et Van Lanen, H. A. J. : Hydrological Drought : Processes and Estimation Methods for Streamflow and Groundwater, Elsevier Edn., 579 pp., 2004.

Toebes, C. and Strang, D. D. : On recession curves - Recession equations, J. Hydrol. NZ, 3, 2-15, 1964.

Zécharias, Y. B. et Brutsaer, W. : Recession characteristics of groundwater outflow and base flow from montainous watersheds, Water Resour. Res., 24, 1651-1658, 1988. 